\title{
Linear scaling solution of the all-electron Coulomb problem in solids
}

\author{
J. E. Pask ${ }^{1, *}$, N. Sukumar ${ }^{2}$ and S. E. Mousavi ${ }^{2}$ \\ ${ }^{1}$ Condensed Matter and Materials Division \\ Lawrence Livermore National Laboratory, Livermore, CA 94550. \\ ${ }^{2}$ Department of Civil and Environmental Engineering \\ University of California, Davis, CA 95616.
}

October 29, 2018

\begin{abstract}
We present a linear scaling formulation for the solution of the all-electron Coulomb problem in crystalline solids. The resulting method is systematically improvable and well suited to large-scale quantum mechanical calculations in which the Coulomb potential and energy of a continuous electronic density and singular nuclear density are required. Linear scaling is achieved by introducing smooth, strictly local neutralizing densities to render nuclear interactions strictly local, and solving the remaining neutral Poisson problem for the electrons in real space. While the formulation includes singular nuclear potentials without smearing approximations, the required Poisson solution is in Sobolev space $H^{1}$, as required for convergence in the energy norm. We employ enriched finite elements, with enrichments from isolated atom solutions, for an efficient solution of the resulting Poisson problem in the interacting solid. We demonstrate the accuracy and convergence of the approach by direct comparison to standard Ewald sums for a lattice of point charges, and demonstrate the accuracy in all-electron quantum mechanical calculations with an application to crystalline diamond.
\end{abstract}

\section{Introduction}

The evaluation of the electrostatic potential and total energy of crystalline solids has been an ongoing problem since the earliest days of solid state physics (Madelung 1918; Ewald 1921; Wigner and Seitz 1933; Fuchs 1935; Ihm et al. 1979; Weinert 1981). In ab initio densityfunctional (Hohenberg and Kohn 1964; Kohn and Sham 1965; Jones and Gunnarsson 1989) calculations, the electrostatic (Coulomb) potential is a sum of nuclear and electronic contributions. In an infinite crystal, however, each of these terms diverges and the sum is

\footnotetext{
*Corresponding author. E-mail: pask1@llnl.gov
} 
only conditionally convergent due to the long-range $1 / r$ nature of the Coulomb interaction. Similarly, the total Coulomb energy is a sum of electron-nucleus, electron-electron, and nucleus-nucleus contributions, each of which diverges in an infinite crystal but combine to yield a finite total electrostatic energy per unit cell. Hence, in the all-electron quantum mechanical problem in solids, there are three distinct divergences which must be addressed simultaneously: (1) the $1 / r$ divergence of the electrostatic potential at the nuclei, (2) the divergence of both potential and energy lattice sums due to the the long-range $1 / r$ nature of the Coulomb interaction, and (3) the infinite self energies of the nuclei.

It has been appreciated for some time that the divergences and conditional convergence of such extended lattice summations can be eliminated by formulating the summations in terms of neutral densities that are well localized in real and/or reciprocal (Fourier) space (Ewald 1921). In the pseudopotential approximation (Pickett 1989), the nuclei and core electrons are replaced by smooth ionic cores, thus eliminating $1 / r$ divergences and infinite self energies. In the conventional reciprocal space approach for such calculations in crystals (Ihm et al. 1979, Pickett 1989), the remaining lattice sum divergences are eliminated by adding neutralizing densities to otherwise divergent Coulomb terms in such a way that the effects of the added densities cancel in the final expressions. Remaining long-range interactions are then rendered short-ranged by transforming to reciprocal space, where smooth periodic functions, of infinite extent in real space, are well localized. However, the resulting expressions for the electrostatic potential and total energy contain structure factors and/or Ewald sums, and require at least $O(N \log N)$ operations to evaluate, where $N$ is the number of atoms in the unit cell. Moreover, since the approach relies on Fourier transforms, it is difficult to implement efficiently on large-scale parallel computational architectures due to the need for extensive interprocessor communications. The limitations of the reciprocal space approach have inspired much research on real-space and local-orbital based approaches (Arias 1999; Beck 2000; Soler et al. 2002; Pask and Sterne 2005a; Torsti et al. 2006), which allow for better scaling, a variety of boundary conditions, and eliminate the need for Fourier transforms. These approaches accomplish a linear scaling solution of the Coulomb problem by solving Poisson's equation in real space, or evaluating the associated integral, thus alleviating the need for Fourier transforms, and allowing the use of efficient multi-level preconditioning (Brandt 1977; Beck 2000).

In the all-electron quantum mechanical context, however, the divergences of the nuclear potentials and self-energies must be confronted in addition to the divergences in lattice sums. Moreover, the rapid, local variation of the electronic density and potential in the vicinity of the nuclear singularities must be addressed. One approach to dealing with nuclear singularities is to approximate the singular nuclear densities by finite, localized functions, e.g., Gaussians or step functions (Merrick et al. 1995; Modine et al. 1997; Goedecker and Ivanov 1998; Wang and Beck 2000; Suryanarayana et al. 2010). This makes possible the solution of a nonsingular total electrostatic potential, due to both nuclei and electrons, in a single linearly scaling step via solution of Poisson's equation with nonsingular total (nuclear + electronic) density as source term. Furthermore, it makes possible the direct evaluation of the total Coulomb energy, with finite nuclear self-energy that is readily removed. However, as the widths of the model nuclear densities are decreased toward physical nuclear dimensions (on the order of $10^{-5}$ a.u.) to achieve convergence, the potential in the vicinity of the nuclei and nuclear self-energies become correspondingly large, causing greater 
absolute errors, which hinder the computation of accurate energy differences for different configurations. Moreover, finer resolution, and correspondingly more degrees of freedom, are required to resolve the more rapidly varying potential in the vicinity of the nuclei, thus increasing computational cost. This was well demonstrated in a remarkable calculation of the Coulomb potential of a uranium dimer (Goedecker and Ivanov 1998) using a second generation interpolating wavelet basis, wherein 22 levels of refinement were required to reduce the maximum error in the potential to order $10^{-2}$ a.u. These difficulties are a consequence of the divergent limit of the sequence: since the three-dimensional Dirac-delta is not in $H^{-1}$, the corresponding solution of Poisson's equation is not in $H^{1}$, where $H^{m}$ is the Sobolev space of order $m$. Therefore, the Coulomb energy diverges and a convergent approximation in $H^{1}$ cannot be constructed.

Other approaches for all-electron quantum mechanical calculations include singular nuclear contributions analytically and compute the remaining contributions analytically or numerically, depending on the choice of basis. One such approach is to compute the electronic contributions via solution of Poisson's equation with nonsingular, though rapidly varying, electronic density as source term (White et al. 1989; Murakami et al. 1992; Tsuchida and Tsukada 1995, Batcho 2000, Beck 2000; Yamakawa and Hyodo 2005; Torsti et al. 2006, Bylaska et al. 2009; Lehtovaara et al. 2009); where in crystalline calculations (Tsuchida and Tsukada 1995; Beck 2000; Torsti et al. 2006), the density must be neutralized to avoid divergent lattice sums. With mesh refinement (Murakami et al. 1992; Tsuchida and Tsukada 1995; Batcho 2000; Torsti et al. 2006; Bylaska et al. 2009; Lehtovaara et al. 2009) and/or addition of well-chosen localized functions to the basis (Yamakawa and Hyodo 2005), one can then solve the resulting Poisson equation for the electronic contribution to the all-electron potential in a single $O(N)$ scaling step. Integrals involving the singular total (electronic + nuclear) potential can be efficiently computed using a transformation of the singular nuclear part (White et al. 1989; Murakami et al. 1992; Batcho 2000; Yamakawa and Hyodo 2005). However, in the crystalline case (Tsuchida and Tsukada 1995), the remaining nuclear contribution must then be computed by lattice summation, which scales as $O\left(N^{2}\right)$ or $O(N \log N)$ at best. An alternative approach to the calculation of the electronic contribution is direct evaluation of the associated Coulomb law integral (Genovese et al. 2006, Juselius and Sundholm 2007; Watson and Hirao 2008, Losilla et al. 2010). This approach, recently extended to periodic calculations (Losilla et al. 2010), can accommodate a variety of boundary conditions, can attain high accuracy, and when combined with the fast multipole method (Greengard and Rokhlin 1987, Strain et al. 1996) for far-field contributions, can achieve $O(N)$ scaling (Watson and Hirao 2008). However, the approach can be sensitive to the approximation employed for the singular 1/|x $-\mathbf{x}^{\prime} \mid$ kernel (Juselius and Sundholm 2007, Watson and Hirao 2008, Losilla et al. 2010), and as with all such approaches computing just the electronic contribution, it leaves the singular nuclear contribution to be computed by other means.

Another approach, employed in accurate all-electron density-functional electronic structure methods (Singh and Nordstrom 2006), employs a dual representation of the density and potential (Rudge 1969; Weinert 1981; Blöchl 1994; Nikolaev and Dyachkov 2002). In this approach, the unit cell is partitioned into atom-centered sphere and interstitial (between spheres) regions. Inside the spheres, where the potential is singular and most rapidly varying, a radial-angular spherical harmonic representation is employed. Outside the spheres, where the potential is generally smooth, a Fourier representation is used. The all-electron 
potential is then computed in two steps: (1) compute the smooth interstitial potential by standard Fourier techniques, and (2) solve the boundary value problem in each sphere using Green's functions, with boundary values from the previously computed interstitial potential. By virtue of the dual representation, this method can provide highly accurate and efficient solutions. However, due to the reliance on Fourier expansions, the scaling is at best $O(N \log N)$ and efficient parallel implementation is difficult due to the need for extensive interprocessor communications.

Fast multipole methods (Greengard and Rokhlin 1987; Strain et al. 1996) provide an $O(N)$ scaling solution of the Coulomb problem and can accommodate periodic (Challacombe et al. 1997; Kudin and Scuseria 1998; Kudin and Scuseria 2000, Kudin and Scuseria 2004) as well as non-periodic boundary conditions. In the context of a Gaussian representation of the charge density, these have become the method of choice for large-scale calculations, as they can provide high accuracy, with linear scaling and a moderate prefactor, and are well suited to parallel implementation. However, the computational cost increases rapidly for higher-quality basis sets (Kurashige et al. 2007), and due to the reliance on a Gaussian representation, it is not a general purpose method (Genovese et al. 2006).

In this paper, we present a systematically improvable, linear scaling formulation for the solution of the all-electron Coulomb problem in crystalline solids. Linear scaling is achieved by introducing smooth, strictly local neutralizing densities to render nuclear interactions strictly local, and solving the remaining neutral Poisson problem for the electrons in real space. In so doing, the all-electron problem is decomposed into analytic strictly-local nuclear, and numerical long-range electronic parts; with required numerical solution in $H^{1}$ so that convergence is assured and approximation is optimal in the relevant energy norm. Rapid variations in the required neutral electronic potential in the vicinity of the nuclei are efficiently treated by an enriched finite element Poisson solution, using local radial solutions as enrichments, thus allowing a $O(N)$ scaling solution. The formulation is presented in Section 2 and the solution is elaborated in Section 3. Expressions for the Coulomb energy per unit cell, analytically excluding the divergent nuclear self-energy, are derived. In Section 4 , Coulomb potential and energy calculations for two canonical test cases using cubic finite elements (FEs) and enriched finite elements (EFEs) are presented. We demonstrate the accuracy and convergence of the approach by direct comparison to standard Ewald sums for a lattice of point charges, and demonstrate the accuracy in quantum mechanical calculations with an application to crystalline diamond. In Section 5 , the main findings are summarized and the outlook of the proposed formulation in density-functional calculations is indicated.

\section{Formulation}

The total Coulomb potential $V(\mathbf{x})$ diverges at the nuclear positions due to the divergence of the nuclear potentials $V_{i}(\mathbf{x})=\frac{q_{i}}{\left|\mathbf{x}-\boldsymbol{\tau}_{i}\right|}$ of charges $q_{i}$ at positions $\boldsymbol{\tau}_{i}$ in the unit cell (where atomic units are used here and throughout). Moreover, the potentials $V^{+}(\mathbf{x})$ due to all nuclei and $V^{-}(\mathbf{x})$ due to all electrons in the crystal diverge at all points in the cell due to the long-range $1 / r$ nature of the Coulomb interaction. To resolve these divergences, we 


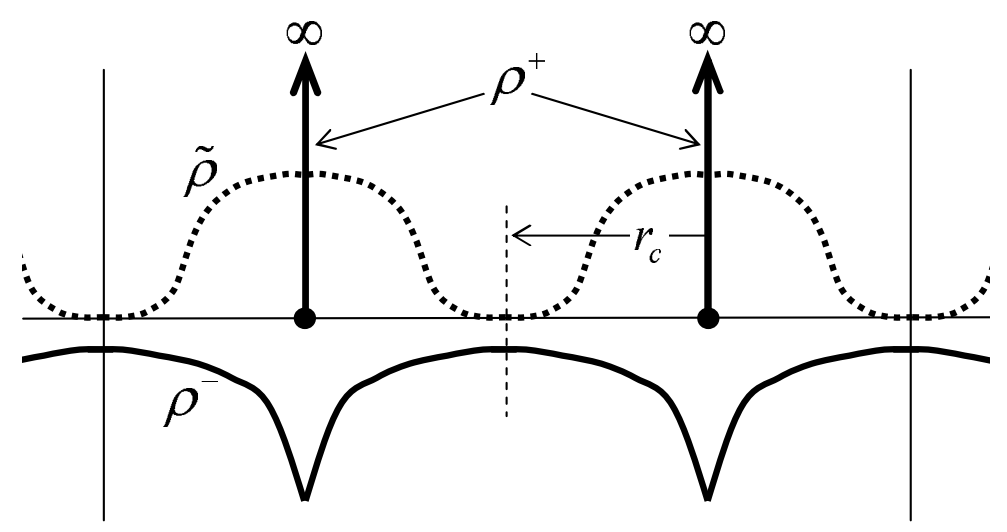

Figure 1: Schematic charge density in unit cell. Total density $\rho=\rho^{+}+\rho^{-}$, the sum of nuclear point charge and continuous electronic densities. Smooth neutralizing density $\tilde{\rho}$ is introduced to render $\rho^{+}$short-ranged and $\rho^{-}$neutral and amenable to direct Poisson solution. $\tilde{\rho}$ is constructed as a sum of smooth, localized densities $\tilde{\rho}_{i}$, strictly local within $r=r_{c}$.

introduce a smooth neutralizing density $\tilde{\rho}$ and write the total charge $\rho$ in the unit cell as

$$
\begin{aligned}
\rho(\mathbf{x}) & =\rho^{+}(\mathbf{x})+\rho^{-}(\mathbf{x}) \\
& =\underbrace{\rho^{+}(\mathbf{x})-\tilde{\rho}(\mathbf{x})}_{\begin{array}{c}
\text { neutralized nuclear } \\
\text { charge density }
\end{array}}+\underbrace{\rho^{-}(\mathbf{x})+\tilde{\rho}(\mathbf{x})}_{\begin{array}{c}
\text { neutralized electronic } \\
\text { charge density }
\end{array}} \\
& =\tilde{\rho}^{+}(\mathbf{x})+\tilde{\rho}^{-}(\mathbf{x}),
\end{aligned}
$$

where $\rho^{+}(\mathbf{x})=\sum_{i} \rho_{i}(\mathbf{x})=\sum_{i} q_{i} \delta\left(\mathbf{x}-\boldsymbol{\tau}_{i}\right)$ is the total nuclear charge density, the sum of nuclear densities in the unit cell, $\rho^{-}(\mathbf{x})$ is the electronic charge density, and $\tilde{\rho}^{+}(\mathbf{x})=$ $\rho^{+}(\mathbf{x})-\tilde{\rho}(\mathbf{x})$ and $\tilde{\rho}^{-}(\mathbf{x})=\rho^{-}(\mathbf{x})+\tilde{\rho}(\mathbf{x})$ are the neutralized nuclear and electronic densities, respectively. The integral over the unit cell $\int_{\Omega} \tilde{\rho}=\sum_{i} q_{i}=Q$, the total nuclear charge in the cell (Fig. 1). In order to produce a linear scaling formulation, we form $\tilde{\rho}$ in the unit cell as a sum of smooth, strictly local densities $\tilde{\rho}_{I}$ centered at atomic positions $\boldsymbol{\tau}_{I}$ with integrals $\int \tilde{\rho}_{I}=q_{I}: \tilde{\rho}=\sum_{I} \tilde{\rho}_{I}$, where the sum is over all sites $I$ in the crystal such that $\tilde{\rho}_{I} \not \equiv 0$ (i.e. $\tilde{\rho}_{I}$ is nonvanishing) in the unit cell. Since the $\tilde{\rho}_{I}$ are strictly localized within a given radius $r=r_{c}$ of each site $I$, i.e., $\tilde{\rho}_{I}(\mathbf{x})=0$ for $\left|\mathbf{x}-\boldsymbol{\tau}_{I}\right|>r_{c}$, the number of terms in the sum varies linearly with the number of atoms the unit cell.

The total potential $V(\mathbf{x})$ in the unit cell may now be written as

$$
V(\mathbf{x})=V^{+}(\mathbf{x})+V^{-}(\mathbf{x})=\tilde{V}^{+}(\mathbf{x})+\tilde{V}^{-}(\mathbf{x}),
$$

the sum of potentials corresponding to neutralized nuclear and electronic densities $\tilde{\rho}^{+}(\mathbf{x})$ and $\tilde{\rho}^{-}(\mathbf{x})$. Let $V_{I}(\mathbf{x})=\frac{q_{I}}{\left|\mathbf{x}-\tau_{I}\right|}$ and $\tilde{V}_{I}(\mathbf{x})$ be the potentials corresponding to charge densities $\rho_{I}(\mathbf{x})=q_{I} \delta\left(\mathbf{x}-\boldsymbol{\tau}_{I}\right)$ and $\tilde{\rho}_{I}(\mathbf{x})$ at each site $I$ and let $\tilde{\rho}_{I}(\mathbf{x})$ be spherically symmetric. Then both potential $V_{I}-\tilde{V}_{I}$ and corresponding neutralized density $\rho_{I}-\tilde{\rho}_{I}$ vanish beyond $r=r_{c}$ about site $I$, since $\tilde{V}_{I}=q_{I} / r$ for $r \geq r_{c}$ due to the compact-support of the associated spherically symmetric $\tilde{\rho}_{I}(\mathbf{x})$. The total potential in the unit cell associated with neutralized nuclear density $\tilde{\rho}^{+}$may then be computed as a short-ranged sum in real space:

$$
\tilde{V}^{+}(\mathbf{x})=\sum_{I} V_{I}(\mathbf{x})-\tilde{V}_{I}(\mathbf{x})=\sum_{I} \frac{q_{I}}{\left|\mathbf{x}-\boldsymbol{\tau}_{I}\right|}-\tilde{V}_{I}(\mathbf{x}),
$$


where the sum is over all sites $I$ in the crystal such that $V_{I}(\mathbf{x})-\tilde{V}_{I}(\mathbf{x}) \not \equiv 0$ in the unit cell. Due to the strict locality of $V_{I}(\mathbf{x})-\tilde{V}_{I}(\mathbf{x})$ around site $I$, the number of nonzero terms in the sum varies linearly with number of atoms in the unit cell.

The potential associated with neutralized electronic density $\tilde{\rho}^{-}$can be obtained from a solution of Poisson's equation:

$$
\nabla^{2} \tilde{V}^{-}(\mathbf{x})=-4 \pi \tilde{\rho}^{-}(\mathbf{x})
$$

subject to periodic boundary conditions, with continuous neutralized electronic density $\tilde{\rho}^{-}(\mathbf{x})$ as source term. At this point, we note that by virtue of the decomposition (1), the source term $\tilde{\rho}^{-}$in (4), unlike the total charge density $\rho$, is in $H^{-1}$ (indeed, it is $C^{0}$ ); so that the corresponding solution $\tilde{V}^{-}$is in $H^{1}$, thus allowing convergent and optimal approximation in the energy norm (Strang and Fix 1973). Moreover, the solution can be accomplished in $O(N)$ operations in real space (Beck 2000), where $N$ is the number of atoms in the unit cell. The total all-electron Coulomb potential $V(\mathbf{x})$ can thus be obtained in $O(N)$ operations using decompositions (11) and (2), without distributed nucleus or other approximations.

The total Coulomb energy per unit cell in the all-electron case is divergent due to the divergence of the nuclear self energies. Thus, the desired total Coulomb energy excluding nuclear self-energy cannot be computed as in the pseudopotential case (Pask and Sterne 2005b) by first computing the total Coulomb energy and then subtracting the self-energy. Instead, the divergent nuclear self-energy must be excluded analytically.

The total Coulomb energy per unit cell can be expressed in terms of densities and associated potentials as

$$
\begin{aligned}
E & =\frac{1}{2} \int_{\Omega} d^{3} x \rho(\mathbf{x}) V(\mathbf{x}) \\
& =\frac{1}{2} \int_{\Omega} d^{3} x\left(\tilde{\rho}^{+}(\mathbf{x})+\tilde{\rho}^{-}(\mathbf{x})\right)\left(\tilde{V}^{+}(\mathbf{x})+\tilde{V}^{-}(\mathbf{x})\right) \\
& =\frac{1}{2} \int_{\Omega} d^{3} x\left(\tilde{\rho}^{+}(\mathbf{x}) \tilde{V}^{+}(\mathbf{x})+2 \tilde{\rho}^{+}(\mathbf{x}) \tilde{V}^{-}(\mathbf{x})+\tilde{\rho}^{-}(\mathbf{x}) \tilde{V}^{-}(\mathbf{x})\right) \\
& =E^{++}+E^{+-}+E^{--},
\end{aligned}
$$

where $E^{++}, E^{+-}$, and $E^{--}$correspond to the $\tilde{\rho}^{+} \tilde{V}^{+}, \tilde{\rho}^{+} \tilde{V}^{-}$, and $\tilde{\rho}^{-} \tilde{V}^{-}$integrals, respectively, and the $\tilde{\rho}^{+} \tilde{V}^{-}$interaction term has been retained in favor of the equivalent $\tilde{\rho}^{-} \tilde{V}^{+}$term to facilitate subsequent integration. The divergent self-energy is contained in the $E^{++}$term. This may be extracted as follows:

$$
E^{++}=\frac{1}{2} \int_{\Omega} d^{3} x \tilde{\rho}^{+}(\mathbf{x}) \tilde{V}^{+}(\mathbf{x})=\frac{1}{2} \int_{\Omega} d^{3} x \sum_{I}\left(\rho_{I}(\mathbf{x})-\tilde{\rho}_{I}(\mathbf{x})\right) \sum_{J}\left(V_{J}(\mathbf{x})-\tilde{V}_{J}(\mathbf{x})\right),
$$

where the sums are over all atomic positions in the crystal with localized densities and potentials $\tilde{\rho}_{I}$ and $V_{J}-\tilde{V}_{J}$ nonzero in the unit cell. Now, we restrict the neutralizing densities $\tilde{\rho}_{I}$ to be nonoverlapping, i.e., $\tilde{\rho}_{I}(\mathbf{x}) \tilde{\rho}_{J}(\mathbf{x})=0$ for $I \neq J$. Then the double summation (66) 
reduces to

$$
\begin{aligned}
E^{++} & =\frac{1}{2} \int_{\Omega} d^{3} x \sum_{I}\left(\rho_{I}(\mathbf{x})-\tilde{\rho}_{I}(\mathbf{x})\right)\left(V_{I}(\mathbf{x})-\tilde{V}_{I}(\mathbf{x})\right) \\
& =\frac{1}{2} \sum_{i} \int d^{3} x\left(\rho_{i}(\mathbf{x})-\tilde{\rho}_{i}(\mathbf{x})\right)\left(V_{i}(\mathbf{x})-\tilde{V}_{i}(\mathbf{x})\right)
\end{aligned}
$$

the sum of neutralized nuclear self energies, where the last summation is over atoms in the unit cell and the integral is over all space. Extracting the divergent self-energy from (17), we have

$$
\begin{aligned}
E^{++} & =E_{\text {self }}-\frac{1}{2} \sum_{i} \int d^{3} x\left[\rho_{i}(\mathbf{x}) \tilde{V}_{i}(\mathbf{x})+\tilde{\rho}_{i}(\mathbf{x})\left(V_{i}(\mathbf{x})-\tilde{V}_{i}(\mathbf{x})\right)\right] \\
& =E_{\text {self }}-\frac{1}{2} \sum_{i}\left[q_{i} \tilde{V}_{i}\left(\boldsymbol{\tau}_{i}\right)+\int d^{3} x \tilde{\rho}_{i}(\mathbf{x})\left(V_{i}(\mathbf{x})-\tilde{V}_{i}(\mathbf{x})\right)\right]
\end{aligned}
$$

This can be simplified further by employing a common spherically symmetric neutralizing charge $\tilde{\rho}_{i}$ and corresponding potential $\tilde{V}_{i}$ at each site. Let $\tilde{\rho}_{i}(\mathbf{x})=q_{i} g\left(\left|\mathbf{x}-\boldsymbol{\tau}_{i}\right|\right)$ and $\tilde{V}_{i}(\mathbf{x})=q_{i} v\left(\left|\mathbf{x}-\boldsymbol{\tau}_{i}\right|\right)$, where $g(r)$ is a smooth radial function strictly localized within $r=r_{c}$, with $\int g(|\mathbf{x}|)=1$ and $r_{c}$ such that $\tilde{\rho}_{i}(\mathbf{x})$ are nonoverlapping, and $v(r)$ is the corresponding potential, as in Fig. 2. For $r>r_{c}$ then, $g(r)=0$ and $v(r)=1 / r$. Equation (8) then becomes

$$
\begin{aligned}
E^{++} & =E_{\text {self }}-\frac{1}{2} \sum_{i}\left[q_{i}^{2} v(0)+q_{i}^{2} \int_{0}^{r_{c}} d r 4 \pi r^{2} g(r)(1 / r-v(r))\right] \\
& =E_{\text {self }}-\frac{1}{2} \sum_{i}\left(q_{i}^{2} v(0)+q_{i}^{2} I_{g}\right),
\end{aligned}
$$

where $I_{g}$ is the constant defined by (9a), which depends only on the choice of the radial function $g(r)$. In the present work, we employ a second-derivative continuous $\left(g \in C^{2}\left(\mathbb{R}_{+}\right)\right)$ piecewise polynomial for $g(r)$ :

$$
g(r)= \begin{cases}-21\left(r-r_{c}\right)^{3}\left(6 r^{2}+3 r r_{c}+r_{c}^{2}\right) /\left(5 \pi r_{c}^{8}\right), & 0 \leq r \leq r_{c} \\ 0, & r>r_{c}\end{cases}
$$

for which

$$
v(r)= \begin{cases}\left(9 r^{7}-30 r^{6} r_{c}+28 r^{5} r_{c}^{2}-14 r^{2} r_{c}^{5}+12 r_{c}^{7}\right) /\left(5 r_{c}^{8}\right), & 0 \leq r \leq r_{c} \\ 1 / r & r>r_{c}\end{cases}
$$

and

$$
I_{g}=10976 /\left(17875 r_{c}\right)
$$




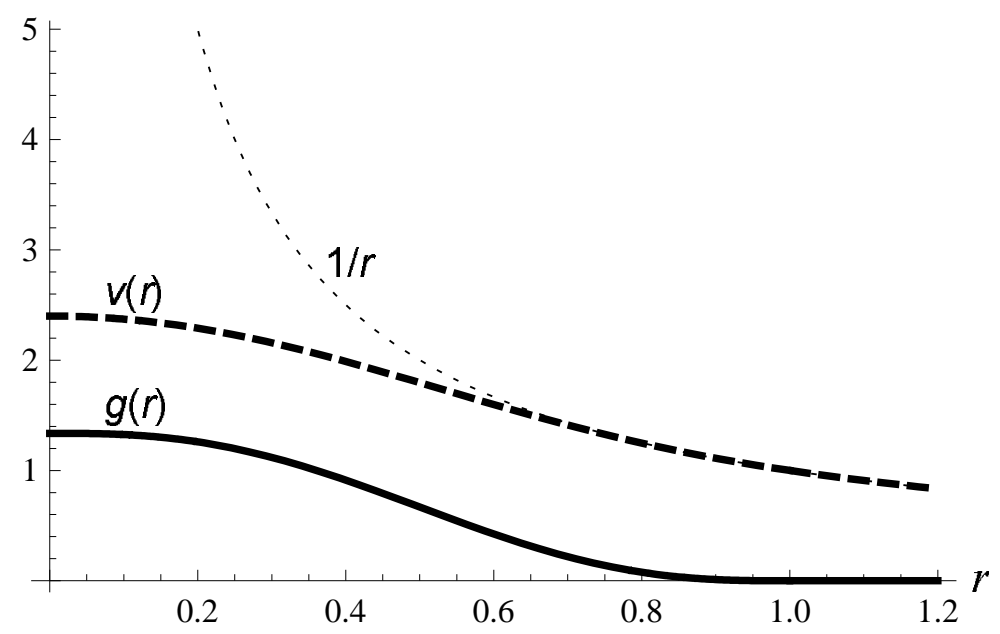

Figure 2: Smooth, strictly local unit charge $g(r)$, corresponding potential $v(r)$, and $1 / r$, with cutoff radius $r_{c}=1 . g(r)=0$ and $v(r)=1 / r$ for $r>r_{c}$.

From $(5 \mathrm{c})$ and $(5 \mathrm{~d})$, the $E^{+-}$interaction term is given by

$$
\begin{aligned}
E^{+-} & =\int_{\Omega} d^{3} x \tilde{\rho}^{+}(\mathbf{x}) \tilde{V}^{-}(\mathbf{x}) \\
& =\int_{\Omega} d^{3} x \sum_{I}\left(\rho_{I}(\mathbf{x})-\tilde{\rho}_{I}(\mathbf{x})\right) \tilde{V}^{-}(\mathbf{x}) \\
& =\sum_{i} q_{i} \tilde{V}^{-}\left(\boldsymbol{\tau}_{i}\right)-\int_{\Omega} d^{3} x \tilde{\rho}(\mathbf{x}) \tilde{V}^{-}(\mathbf{x}),
\end{aligned}
$$

where the sum on $I$ extends over all atoms in the crystal with $\tilde{\rho}_{I} \not \equiv 0$ in the unit cell and the sum on $i$ is over atoms in the unit cell.

Being free of nuclear singularities, the $E^{--}$term can be evaluated straightforwardly as

$$
E^{--}=\frac{1}{2} \int_{\Omega} d^{3} x \tilde{\rho}^{-}(\mathbf{x}) \tilde{V}^{-}(\mathbf{x}) .
$$

Collecting $E^{++}, E^{+-}$, and $E^{--}$from (9b), (11), and (12) above, we arrive at the following expression for the all-electron Coulomb energy per unit cell, excluding nuclear self-energy:

$$
E-E_{\text {self }}=\sum_{i}\left[q_{i} \tilde{V}^{-}\left(\boldsymbol{\tau}_{i}\right)-\frac{1}{2} q_{i}^{2}\left(v(0)+I_{g}\right)\right]+\int_{\Omega} d^{3} x\left(\frac{1}{2} \tilde{\rho}^{-}(\mathbf{x})-\tilde{\rho}(\mathbf{x})\right) \tilde{V}^{-}(\mathbf{x}),
$$

where the integral is over the unit cell and the sum is over atomic positions in the cell. Since the densities and potentials in (13) can be obtained in $O(N)$ operations, as described above, the energy too, as formulated in (131), can be obtained in $O(N)$ operations.

In the above expression, the $\tilde{\rho}^{+} \tilde{V}^{-}$interaction term was retained in favor of the equivalent $\tilde{\rho}^{-} \tilde{V}^{+}$term to facilitate analytic integration. However, whereas this eliminates a threedimensional numerical integration, it produces a term in the energy requiring a pointwise evaluation of the Poisson solution $\tilde{V}^{-}$. In basis-oriented, variational approaches for the Poisson solution, however, energy integrals of the solution converge more rapidly than pointwise 
values (Strang and Fix 1973), and so it is of interest to develop an alternative expression free of pointwise evaluations of $\tilde{V}^{-}$. This can be accomplished at the cost of an additional numerical integration by formulating the $E^{+-}$interaction term based on the corresponding $\tilde{\rho}^{-} \tilde{V}^{+}$integral rather than $\tilde{\rho}^{+} \tilde{V}^{-}$integral as in (5c).

The interaction term is

$$
E^{+-}=\int_{\Omega} d^{3} x \tilde{\rho}^{+}(\mathbf{x}) \tilde{V}^{-}(\mathbf{x})=\int_{\Omega} d^{3} x \tilde{\rho}^{-}(\mathbf{x}) \tilde{V}^{+}(\mathbf{x}) .
$$

This may be expressed as

$$
\begin{aligned}
E^{+-} & =\int_{\Omega} d^{3} x\left[\rho^{-}(\mathbf{x})+\sum_{I} \tilde{\rho}_{I}(\mathbf{x})\right] \sum_{J}\left(V_{J}(\mathbf{x})-\tilde{V}_{J}(\mathbf{x})\right) \\
& =\sum_{I} \int_{\Omega} d^{3} x \rho^{-}(\mathbf{x})\left(V_{I}(\mathbf{x})-\tilde{V}_{I}(\mathbf{x})\right)+\sum_{I} \int_{\Omega} d^{3} x \tilde{\rho}_{I}(\mathbf{x})\left(V_{I}(\mathbf{x})-\tilde{V}_{I}(\mathbf{x})\right) \\
& =\sum_{i} \int d^{3} x \rho^{-}(\mathbf{x})\left(V_{i}(\mathbf{x})-\tilde{V}_{i}(\mathbf{x})\right)+\sum_{i} \int d^{3} x \tilde{\rho}_{i}(\mathbf{x})\left(V_{i}(\mathbf{x})-\tilde{V}_{i}(\mathbf{x})\right)
\end{aligned}
$$

for nonoverlapping densities $\tilde{\rho}_{I}(\mathbf{x})$, where $I$ extends over all contributing sites in the crystal; $i$, over positions in the unit cell, and the final integrals are over all space. On letting $\tilde{\rho}_{i}(\mathbf{x})=q_{i} g\left(\left|\mathbf{x}-\boldsymbol{\tau}_{i}\right|\right)$ and $\tilde{V}_{i}(\mathbf{x})=q_{i} v\left(\left|\mathbf{x}-\boldsymbol{\tau}_{i}\right|\right)$, where $g(r)$ and $v(r)$ are as in (10a) and (10b), the interaction term becomes

$$
E^{+-}=\sum_{i} q_{i} \int_{\Omega_{i}} d^{3} x \rho^{-}(\mathbf{x})\left(1 / r_{i}-v\left(r_{i}\right)\right)+\sum_{i} q_{i}^{2} I_{g}
$$

where $\Omega_{i}$ is the sphere of radius $r=r_{c}$ within which density $\tilde{\rho}_{i}(\mathbf{x})$ is localized, $r_{i}=\left|\mathbf{x}-\boldsymbol{\tau}_{i}\right|$, and $I_{g}$ is as in (10c). With this formulation of the interaction term, the all-electron Coulomb energy per unit cell, excluding nuclear self energy becomes

$$
E-E_{\text {self }}=\sum_{i} \frac{1}{2} q_{i}^{2}\left(I_{g}-v(0)\right)+\sum_{i} q_{i} \int_{\Omega_{i}} d^{3} x \rho^{-}(\mathbf{x})\left(1 / r_{i}-v\left(r_{i}\right)\right)+\frac{1}{2} \int_{\Omega} d^{3} x \tilde{\rho}^{-}(\mathbf{x}) \tilde{V}^{-}(\mathbf{x}) .
$$

In the special case of constant $\rho^{-}(\mathbf{x})$, as in the Ewald problem, the integral in the second term of (17) reduces to $I_{s p h}=14 \rho^{-} \pi r_{c}^{2} / 75$. In the more general case, the weak angular dependence of $\rho^{-}(\mathbf{x})$ about each site $i$ allows for efficient evaluation using Gaussian quadrature in spherical coordinates (Stroud 1971).

\section{Solution}

The computation of the all-electron Coulomb potential and energy as formulated in (2), (13), and (17) requires the solution of the Poisson equation (4) for the potential $\tilde{V}^{-}$corresponding to the neutralized electronic charge density $\tilde{\rho}^{-}$, which may be accomplished by a number of methods. Here, we employ an enriched finite element (EFE) method (Strang and Fix 1973; Melenk and Babuška 1996; Babuška and Melenk 1997; Sukumar and Pask 2009) in order to 
efficiently resolve the sharp variations in the all-electron densities and potentials while retaining systematic improvability and $O(N)$ scaling in the solution process. Note that some form of multilevel preconditioning is generally required to achieve linear scaling of solution time with respect to number of degrees of freedom (DOFs) in FE and related methods. While not demonstrated as yet in the context of EFE methods, to our knowledge, it is expected that such preconditioning will apply here also. EFE methods add fixed enrichment functions to the classical $\mathrm{FE}$ basis in order to efficiently represent rapid variations in the solution. This leaves a smooth residual (difference between exact solution and enrichment) for the remaining FE basis to represent, thus allowing a substantially coarser FE mesh. EFE methods can thus be understood as FE methods on softer problems, and so obtain classical asymptotic convergence rates with respect to mesh size, depending only on the completeness of the classical part of the basis (as shown below). Hence, multilevel preconditioning may be expected to apply to EFE as for FE. Indeed, consistent with this expectation, linear scaling with respect to number of DOFs has been demonstrated recently in the context of partition-of-unity enrichment methods (Schweitzer 2003, Schweitzer 2008).

In the classical FE Poisson solution (Pask and Sterne 2005a), the potential $\tilde{V}^{-}$is expressed as a linear combination of strictly local, piecewise polynomial basis functions $\left\{\phi_{i}\right\}$ :

$$
\tilde{V}_{F E}^{-}(\mathbf{x})=\sum_{i} \phi_{i}(\mathbf{x}) a_{i}^{F E}
$$

In the enriched FE solution, a set of functions $\left\{\psi_{\alpha}\right\}$ which incorporates a priori information about the solution is added to the classical basis in order to substantially reduce the number of basis functions required to attain a given accuracy; so that $\tilde{V}^{-}$is expressed as

$$
\tilde{V}^{-}(\mathbf{x})=\sum_{i} \phi_{i}(\mathbf{x}) a_{i}+\sum_{\alpha} \psi_{\alpha}(\mathbf{x}) b_{\alpha} \equiv \sum_{k} \Phi_{k}(\mathbf{x}) c_{k}
$$

where $\alpha$ is summed over the atoms and $\left\{\Phi_{k}\right\}=\left\{\phi_{i}\right\} \cup\left\{\psi_{\alpha}\right\}$ is the enriched FE basis, the combined set of classical $\mathrm{FE}$ and enrichment basis functions.

In the present case, the desired solution $\tilde{V}^{-}=V^{-}+\tilde{V}$ is the potential associated with neutralized electronic charge density $\tilde{\rho}^{-}=\rho^{-}+\tilde{\rho}$, where $\tilde{\rho}=\sum_{I} \tilde{\rho}_{I}$, the sum of smooth, strictly local, nonoverlapping neutralizing densities over atomic sites $I$ in the crystal such that $\tilde{\rho}_{I} \not \equiv 0$ in the unit cell. In the all-electron case, the electronic density $\rho^{-}$is large in magnitude, rapidly varying, and isolated-atom-like in the vicinity of the nuclei while much smaller in magnitude and more smoothly varying elsewhere. The neutralizing density $\tilde{\rho}$ is moderate in magnitude and smoothly varying throughout the cell. Hence, the rapid variations in the desired solution $\tilde{V}^{-}$associated with neutralized electronic density $\tilde{\rho}^{-}$are confined to the vicinity of the nuclei where the electronic density $\rho^{-}$is large, rapidly varying, and atomic-like. To increase the efficiency of the representation, therefore, we might add to the basis the potentials $\tilde{v}_{I}^{-}$corresponding to the neutralized electronic densities $\tilde{\rho}_{I}^{-}=\rho_{I}^{-}+\tilde{\rho}_{I}$ in the vicinity of each site $I$; where the local electronic densities $\rho_{I}^{-}$vary like the total electronic density $\rho^{-}$in the vicinity of site $I$ and integrate to the appropriate charge $-q_{I}$. These may be obtained, for example, from a partitioning of the self-consistent electronic density or from isolated atom densities. 
For each atom $\alpha$ in the unit cell, then, an enrichment function

$$
\tilde{V}_{\alpha}^{-}(\mathbf{x})=\sum_{\mathbf{R}} \tilde{v}_{\alpha}^{-}(\mathbf{x}-\mathbf{R})
$$

approximating the desired solution $\tilde{V}^{-}$in the vicinity of atom $\alpha$ is constructed, where $\mathbf{R}$ denotes lattice translation vectors such that $\tilde{v}_{\alpha}^{-}(\mathbf{x}-\mathbf{R}) \not \equiv 0$ in the unit cell. Since the enrichment is only needed in the vicinity of atom $\alpha$, the $\tilde{v}_{\alpha}^{-}$and associated summation are short-ranged. The enriched basis functions $\psi_{\alpha}$ in (19) are taken, then, as the periodic enrichment functions $\tilde{V}_{\alpha}^{-}$:

$$
\psi_{\alpha}(\mathbf{x})=\tilde{V}_{\alpha}^{-}(\mathbf{x}) .
$$

To solve the Poisson equation subject to periodic boundary conditions, it is sufficient that the basis $\left\{\Phi_{k}\right\}$ satisfy

$$
\Phi_{k}(\mathbf{x}+\mathbf{R})=\Phi_{k}(\mathbf{x})
$$

for all $\mathbf{x}$ on the boundary (Pask et al. 1999; , Pask and Sterne 2005a). Classical basis functions $\left\{\phi_{i}\right\}$ satisfying this condition are readily constructed (Pask et al. 1999). Furthermore, the enriched basis functions $\left\{\psi_{\alpha}\right\}$ in (21) are periodic in the unit cell by construction (Eq. (20) ). Thus the enriched FE basis $\left\{\Phi_{k}\right\}$ as a whole satisfies the required condition also (Sukumar and Pask 2009).

Having so constructed the enriched basis $\left\{\Phi_{j}\right\}$, satisfying the required boundary conditions, the enriched FE solution $\tilde{V}^{-}(\mathbf{x})=\sum_{j} \Phi_{j}(\mathbf{x}) c_{j}$ of the Poisson equation (4) with neutralized electronic density $\tilde{\rho}^{-}(\mathbf{x})$ is then determined by the sparse, symmetric linear system (Pask and Sterne 2005a)

$$
\sum_{j} L_{i j} c_{j}=f_{i}
$$

where

$$
\begin{aligned}
L_{i j} & =\int_{\Omega} d^{3} x \nabla \Phi_{i}(\mathbf{x}) \cdot \nabla \Phi_{j}(\mathbf{x}) \\
f_{i} & =4 \pi \int_{\Omega} d^{3} x \Phi_{i}(\mathbf{x}) \tilde{\rho}^{-}(\mathbf{x})
\end{aligned}
$$

\section{Results}

We demonstrate the accuracy and convergence of the formulation presented in Section 3 on two canonical test cases: the Ewald energy of a bcc crystal, for which a reference value is available via standard Ewald summation (Ewald 1921), and the all-electron Coulomb potential and energy of crystalline diamond. In the numerical computations, the parallelepiped unit cell is discretized into regular $m \times m \times m$ serendipity cubic (32-node) finite elements. The shape function expressions for serendipity cubic brick elements are provided in Zienkiewicz et al. (2005), Upon obtaining radial atomic potential solutions $v_{\alpha}^{-}$analytically or from a one-dimensional solver (exploiting spherical symmetry), the neutralized atomic potential functions $\tilde{v}_{\alpha}^{-}$are tabulated on a one-dimensional equi-spaced grid for the 
Ewald problem and on a logarithmic grid for the diamond problem. A quintic spline-fit of the tabulated data is formed and the enrichment function is constructed as in (20), which is then used in the numerical computations. Numerical integration for the FE computations is carried out using $5 \times 5 \times 5$ Gauss quadrature, and higher-order quadrature is employed in EFE computations. The FE computation on a $m \times m \times m$ mesh has $7 m^{3}$ degrees of freedom, and the corresponding EFE computation has just two (number of atoms) more.

\subsection{Ewald problem}

For the classical Ewald energy, we consider a reference bcc crystal with unit atomic spacing defined by lattice vectors

$$
\begin{aligned}
& \mathbf{a}_{1}=a(1,0,0), \\
& \mathbf{a}_{2}=a(0,1,0), \\
& \mathbf{a}_{3}=a(0,0,1),
\end{aligned}
$$

with unit point charges $q_{i}=1$ located at positions (in lattice coordinates)

$$
\begin{aligned}
& \boldsymbol{\tau}_{1}=(0,0,0), \\
& \boldsymbol{\tau}_{2}=(1 / 2,1 / 2,1 / 2)
\end{aligned}
$$

and a constant negative background $\rho^{-}=-2 / a^{3}$ bohr $^{-3}$, where $a=2 / \sqrt{3}$ bohr. The Ewald energy computed by standard $N^{2}$ scaling summation is -1.5758343085 Hartree/atom.

To compute the energy using the $O(N)$ formulation (13) or (17), we take $g(r)$ as in (10a) with $r_{c}=1 / 2$ bohr, so that the resulting neutralizing densities $\tilde{\rho}_{I}(\mathbf{x})=g\left(\left|\mathbf{x}-\boldsymbol{\tau}_{I}\right|\right)$ at each site $I$ in the crystal are as smooth as possible without overlapping. The total neutralizing density in the cell is then $\tilde{\rho}(\mathbf{x})=\sum_{I} \tilde{\rho}_{I}(\mathbf{x})$, where the sum over positions $I$ in the unit and nearest neighboring cells is sufficient due to the short-range of $g(r)$. For the sake of clarity, we distinguish between the cut-off radius $r_{c}$ used to represent the neutralizing charge $\tilde{\rho}_{I}$ and that used to form the enrichment function corresponding to the neutralized electronic charge density. We refer to the former as $r_{c n}$ and to the latter as $r_{c e}$. Since the electronic density $\rho^{-}$is constant, we take as local electronic densities $\rho_{I}^{-}(\mathbf{x})=-g\left(\left|\mathbf{x}-\boldsymbol{\tau}_{I}\right|\right)$ with $r_{c e}=1$ a.u. so that $\sum_{I} \rho_{I}^{-}$approximates the constant $\rho^{-}$in the cell with $I$ running again over positions in the unit cell as well as the nearest neighboring cells. The potentials corresponding to neutralized electronic densities $\tilde{\rho}_{I}^{-}=\rho_{I}^{-}+\tilde{\rho}_{I}$ at each site $I$ are then $\tilde{v}_{I}^{-}=v_{I}^{-}+\tilde{v}_{I}$, where $v_{I}^{-}(\mathbf{x})=-v\left(\left|\mathbf{x}-\boldsymbol{\tau}_{I}\right|\right)$ with $r_{c e}=1$ a.u., $\tilde{v}_{I}(\mathbf{x})=v\left(\left|\mathbf{x}-\boldsymbol{\tau}_{I}\right|\right)$ with $r_{c n}=1 / 2$ a.u., and $v(r)$ is as in (10b). The enrichment functions $\tilde{V}_{\alpha}^{-}$are then formed as in (20). The required potential $\tilde{V}^{-}$corresponding to neutralized electronic density $\tilde{\rho}^{-}=\rho^{-}+\tilde{\rho}$ is obtained from the enriched FE solution of the associated Poisson equation subject to periodic boundary conditions, with enrichment functions $\tilde{V}_{\alpha}^{-}$. The total (electronic + nuclear) potential is then obtained from (2) and the energy, from (13) or (17).

The numerical results for the Ewald problem are shown in Figure 3. The electronic, neutralizing, and neutralized electronic charge densities are shown in Figure 3a. The FE potential solution corresponding to the neutralized electronic charge density is plotted along the diagonal in Figure 3b for different meshes; convergence is observed as the number of 
elements along each coordinate direction increases from 3 to 5 to 7 . The two enrichment functions, one for each atom, are shown in Figure 3r, and the EFE solution for a $12 \times 12 \times 12$ mesh is depicted in Figure $3 \mathrm{~d}$. The total potential solution, which includes the singular nuclear potential contributions, is shown in Figure 3e. Figure 4 a shows the convergence curves for the Coulomb energy per atom for FE and EFE solutions. Numerical integration in the EFE solution is carried out with a $20 \times 20 \times 20$ Gauss quadrature rule in each element, which ensures that the quadrature error is less than the approximation error. From Figure 4 a, we see that use of the pointwise expression (13) adversely affects both accuracy and rate of convergence. To explore this further, the pointwise error $\sum_{I=1}^{2} \tilde{V}^{-}\left(\tau_{I}\right)$ is plotted in Figure $4 \mathrm{~b}$, where the EFE solution $(16 \times 16 \times 16$ mesh $)$ is used as reference. Clearly, the pointwise error is appreciable, which explains the decrease in accuracy and the non-monotonic convergence in the curves plotted in Figure 4a. The enriched finite element solution provides an accuracy of order $10^{-8} \mathrm{Ha}$ in the Coulomb energy with 9 elements in each direction whereas with finite elements, 32 elements in each direction is required for the same accuracy. On using the integral expression in (17) to compute the Coulomb energy, the optimal sextic rate of convergence is obtained with FE and EFE (Figure 4a), consistent with theory (Strang and Fix 1973).

From the error curve for EFE in Figure 4 a (integral expression), we note that the mesh $4 \times 4 \times 4$ delivers much better accuracy and convergence rate than the other meshes. Further analysis helps to explain this anomaly. For the Ewald problem, the corner atom is at the origin and the center atom is at $\boldsymbol{\tau}=(1 / 2,1 / 2,1 / 2)$ (length of the diagonal is 2 a.u.), and $r_{c n}=1 / 2$ a.u. ensures no overlap between the neutralizing densities of the two atoms. Furthermore, the support of the neutralizing charges coincides with the location of the nodes $(4 \times 4 \times 4 \mathrm{mesh})$ along the diagonal of the cube. We repeat the calculations for two more cases: $r_{c n}=1 / 3$ a.u., $r_{c e}=1$ a.u.; and $r_{c n}=1 / 3$ a.u., $r_{c e}=2 / 3$ a.u. The results obtained for all three cases are shown in Figure 5 . One can observe that the solutions for the mesh $4 \times 4 \times 4$ with $r_{c n}=1 / 3$ a.u. are consistent with the solutions on other meshes, and hence the markedly better accuracy for the case when $r_{c n}=1 / 2$ a.u. (Figures 4 a and 5 ) is a consequence of the choice $r_{c n}=1 / 2$ a.u.

Numerical integration to compute the weak form integrals is carried out using standard tensor-product Gauss quadrature. Since the right-hand-side of the Poisson equation (4) is not a polynomial, the degree of the quadrature rule must be selected so that the integration error is at least an order smaller than the approximation error in order to obtain variational results. Figure 6] shows the error in the Coulomb energy per atom $(24 \times 24 \times 24 \mathrm{FE}$ mesh $)$ as a function of the number of quadrature points in each direction. The error in the FE solution is shown by the horizontal lines, and Figure 6 reveals that at least five points $(5 \times 5 \times 5$ quadrature rule) are needed to ensure that quadrature error is below approximation error.

\subsection{Diamond}

We now consider diamond, with unit cell defined by lattice vectors

$$
\begin{aligned}
& \mathbf{a}_{1}=a / 2(0,1,1), \\
& \mathbf{a}_{2}=a / 2(1,0,1), \\
& \mathbf{a}_{3}=a / 2(1,1,0)
\end{aligned}
$$




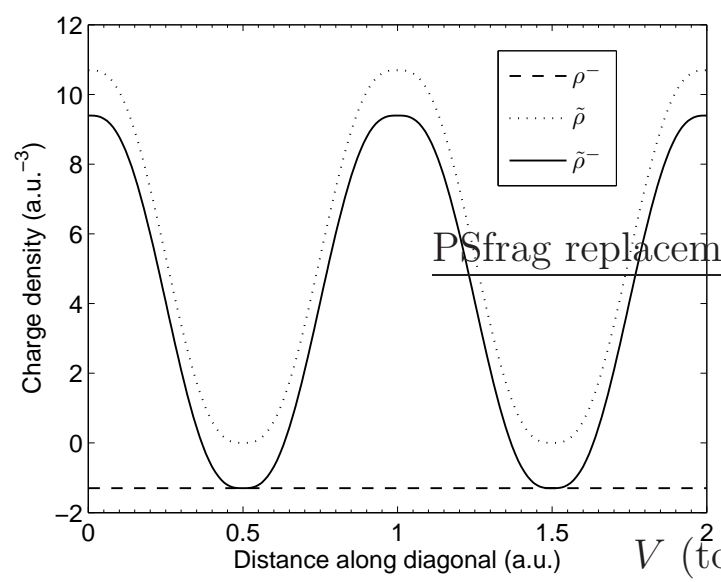

(a)

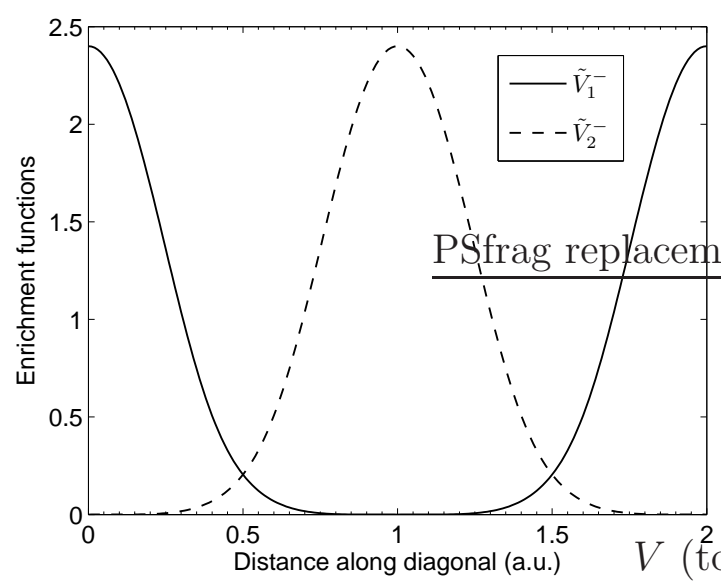

(c)

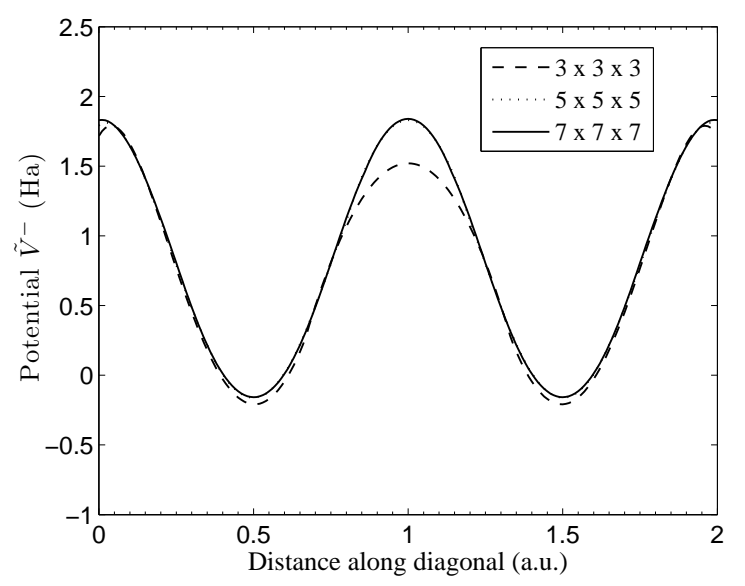

(b)

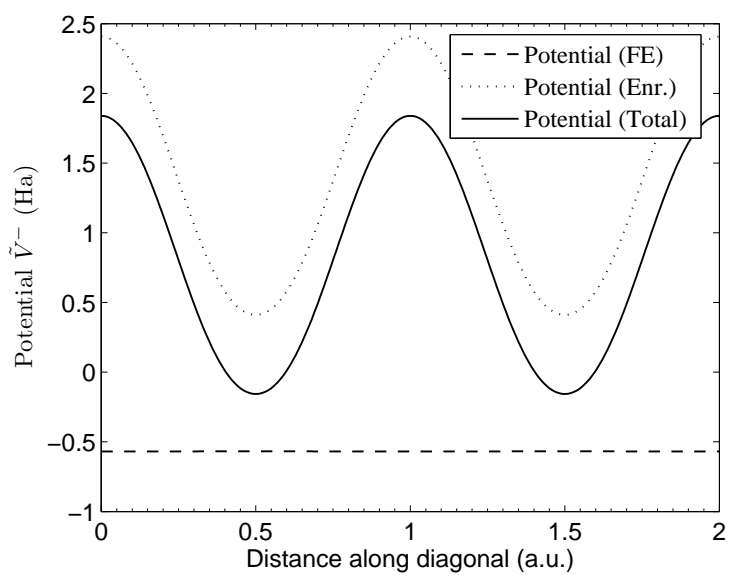

(d)

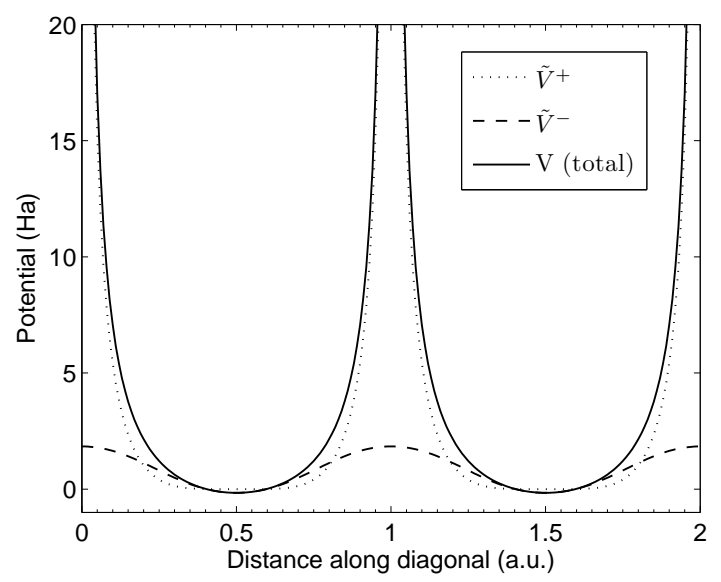

(e)

Figure 3: Finite element (FE) and enriched finite element (EFE) solutions for the Ewald problem. (a) Charge densities; (b) FE solutions; (c) Enrichment functions; (d) EFE solution $(12 \times 12 \times 12 \mathrm{mesh})$; and (e) Total EFE solution $(12 \times 12 \times 12 \mathrm{mesh})$. 


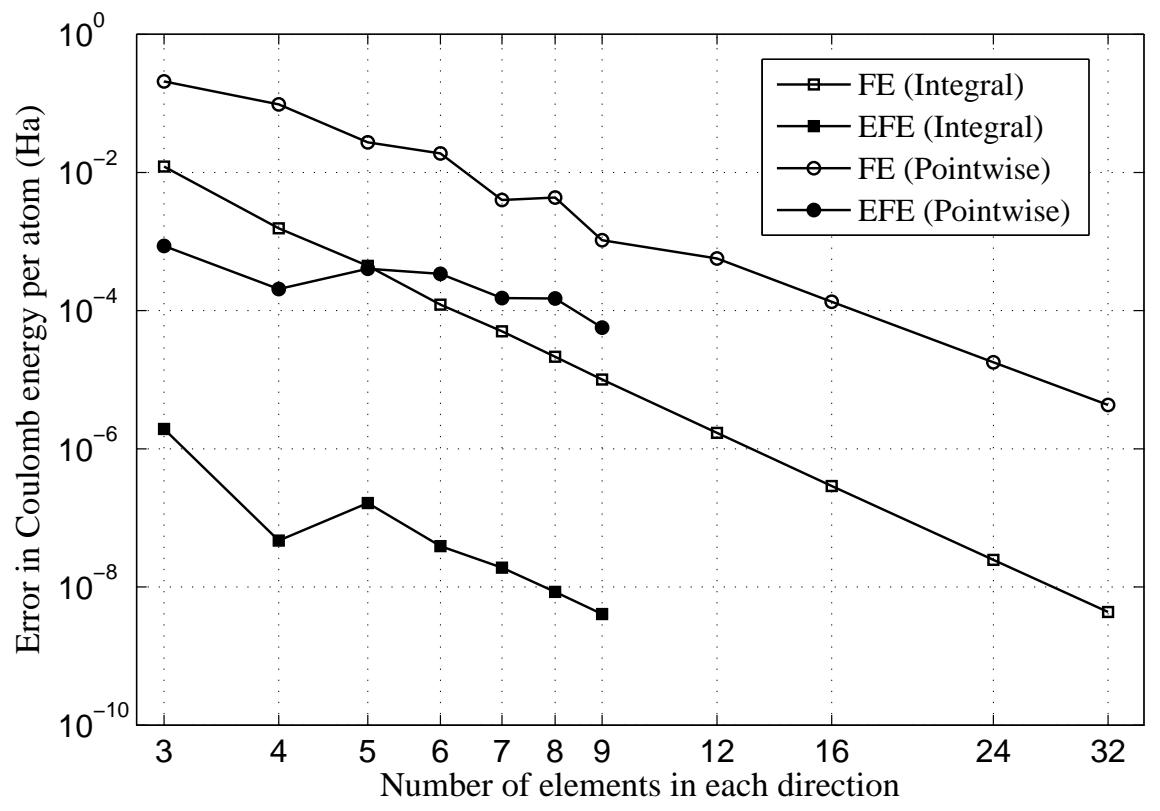

(a)

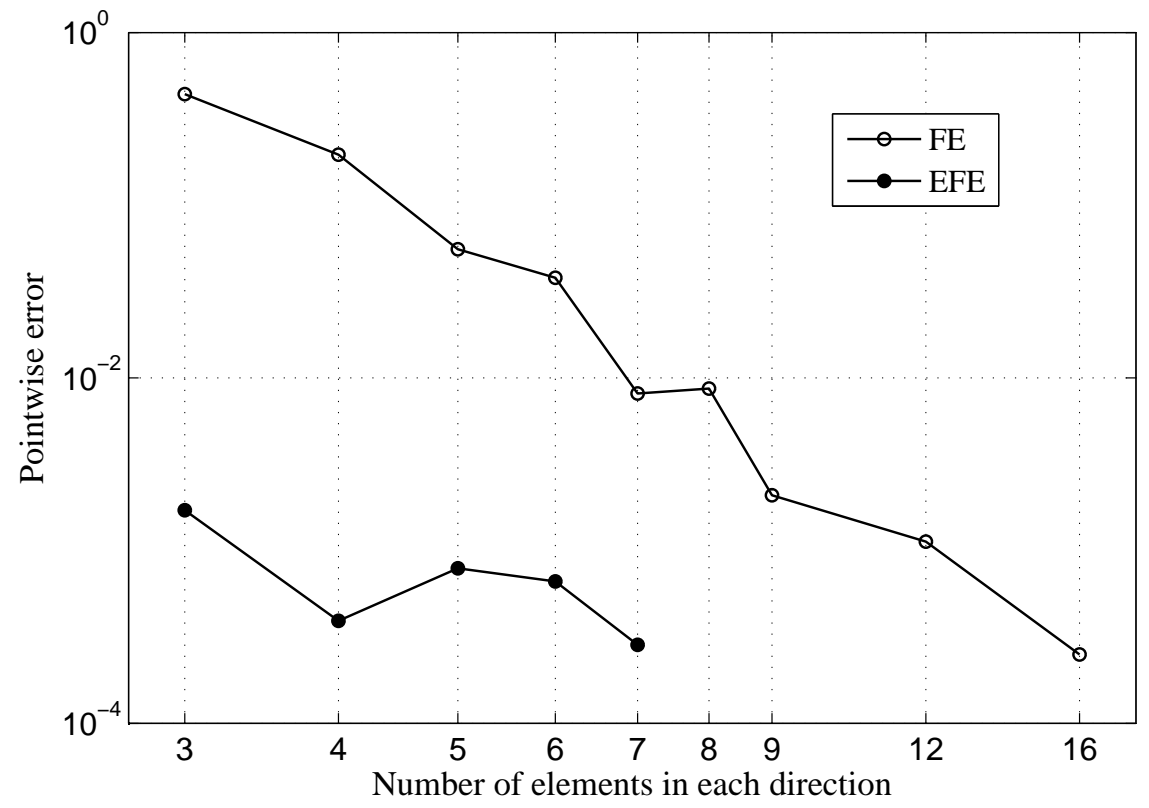

(b)

Figure 4: Convergence curves. (a) Error in Coulomb energy per atom. Convergence rates: $\mathrm{FE}$ (integral) is 6.06, EFE (integral) is 6.15, $\mathrm{FE}$ (pointwise) is 4.97, and EFE (pointwise) is 3.83. (b) Error in $\sum_{I=1}^{2} \tilde{V}^{-}\left(\tau_{I}\right)$. EFE solution on $16 \times 16 \times 16$ mesh is used as reference. 


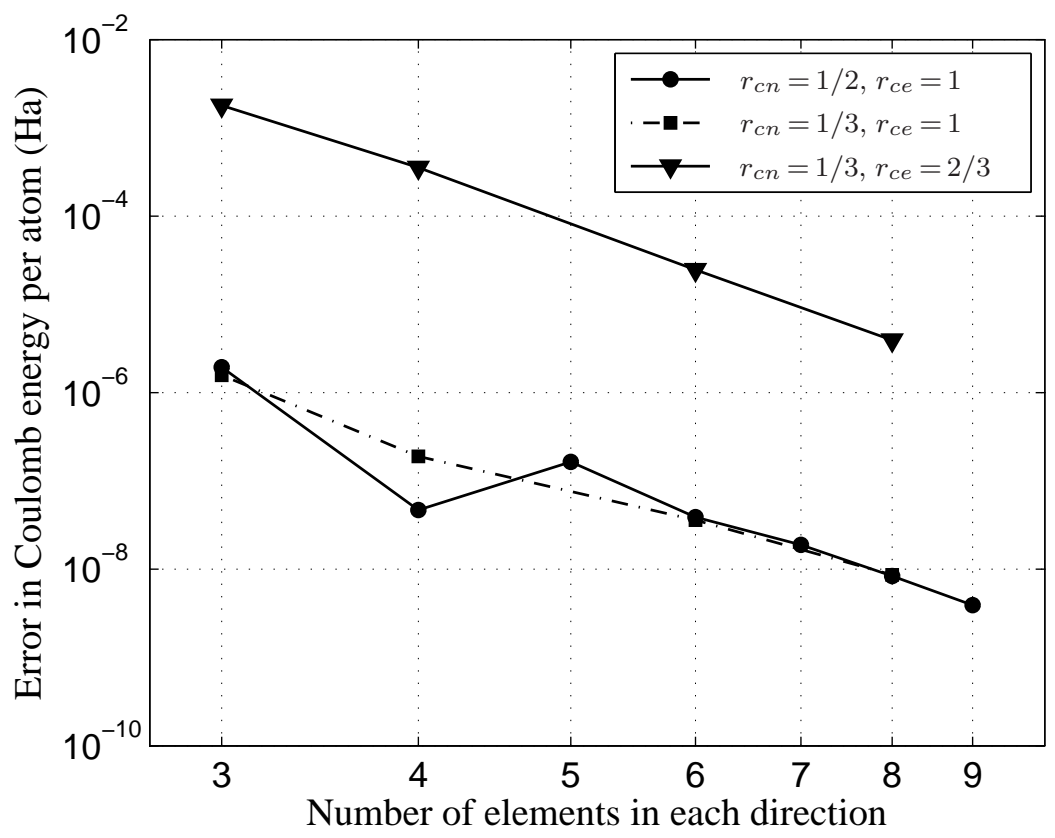

Figure 5: Error in Coulomb energy per atom for EFE computations using different $r_{c n}$ and $r_{c e}$.

and carbon atoms at positions

$$
\begin{aligned}
& \boldsymbol{\tau}_{1}=(0,0,0), \\
& \boldsymbol{\tau}_{2}=(1 / 4,1 / 4,1 / 4),
\end{aligned}
$$

where $a=6.75$ bohr. For the all-electron problem, we consider a total charge density $\rho$ consisting of nuclear point charges $q_{i}=6$ at positions $\boldsymbol{\tau}_{i}$ and electronic density $\rho^{-}$from overlapping all-electron atomic densities.

To compute the energy using the formulation (13) or (17), we take $g(r)$ as in (10a) with $r_{c}=1.4$ a.u., so that the neutralizing densities $\tilde{\rho}_{I}(\mathbf{x})=6 g\left(\left|\mathbf{x}-\boldsymbol{\tau}_{I}\right|\right)$ are nonoverlapping. The total neutralizing density in the cell is $\tilde{\rho}(\mathbf{x})=\sum_{I} \tilde{\rho}_{I}(\mathbf{x})$, where the sum over positions $I$ in the unit and nearest neighboring cells is sufficient. The electronic charge density is $\rho^{-}=\sum_{I} \rho_{I}^{-}$, which is the sum of all-electron atomic densities, where the sum over positions in nearest and second-nearest neighbor cells is sufficient to capture the tails of the $\rho_{I}^{-}$. The potentials corresponding to neutralized electronic densities $\tilde{\rho}_{I}^{-}=\rho_{I}^{-}+\tilde{\rho}_{I}$ at each site $I$ are then $\tilde{v}_{I}^{-}=v_{I}^{-}+\tilde{v}_{I}$, where $v_{I}^{-}$is the all-electron atomic potential and $\tilde{v}_{I}$ is the potential corresponding to $\tilde{\rho}_{I}$. The integral in the second term of (17) involving $\rho^{-}(\mathbf{x})$ is computed within a sphere of radius $r_{c}$ using Gauss quadrature in spherical coordinates. A tensorproduct quadrature rule with 531 points (spline-fit has 177 knots) in the radial direction, and 20 points in each of the two angular directions is used. We obtain $I_{s p h}=-9.44150186$ with precision to all digits shown. The enrichment functions $\tilde{V}_{\alpha}^{-}$are formed as in (20), with the $\tilde{v}_{I}^{-}$brought smoothly to zero at radius $a / \sqrt{3}$ in order to maximize their extents consistent with summation over unit and nearest neighbor cells only. The required potential $\tilde{V}^{-}$corresponding to the neutralized electronic density $\tilde{\rho}^{-}$is then obtained from the enriched 


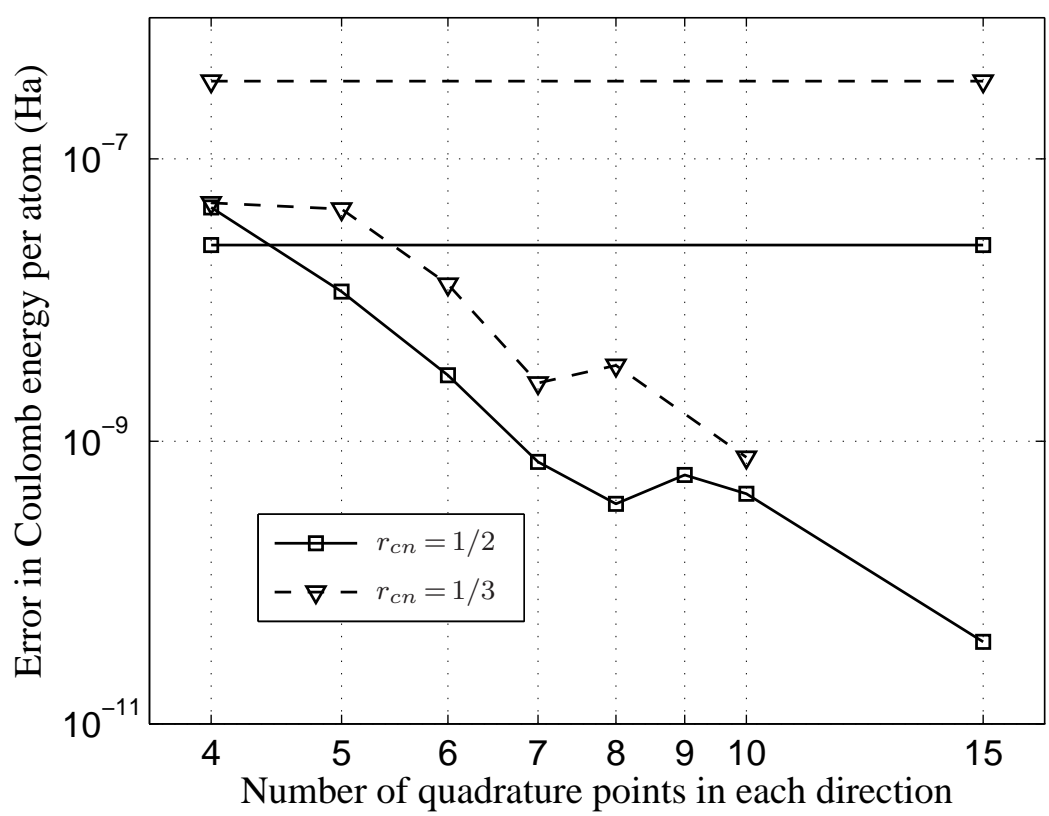

Figure 6: Comparison of quadrature errors for FE computations on $24 \times 24 \times 24$ mesh. Horizontal lines show the best solution error obtained for each case.

FE solution of the associated Poisson equation, with enrichment functions $\tilde{V}_{\alpha}^{-}$; whereupon the Coulomb potential and energy are obtained from (2) and (13) or (17), respectively.

The electronic, neutralizing, and neutralized electronic charge densities are shown in Figure 7 a. The neutralized finite element potential solutions along the diagonal of the unit cell are plotted in Figure $7 \mathrm{~b}$ for 8, 16, and 32 elements along each primitive lattice direction. The two enrichment functions, one for each atom, are shown in Figure [7, and the neutralized EFE potential solution and total potential solution for the $24 \times 24 \times 24$ mesh are illustrated in Figures $7 \mathrm{~d}$ and $7 \mathrm{~b}$. The error in the Coulomb energy with mesh refinement is plotted in Figure 7f for FE and EFE methods; with EFE result on a $24 \times 24 \times 24$ mesh as reference. The enriched finite element solution has an accuracy of $4 \times 10^{-6} \mathrm{Ha}$ in the Coulomb energy for a $16 \times 16 \times 16$ mesh $(28,674$ degrees of freedom), whereas the the best uniform-mesh FE result provides an accuracy of only $6 \times 10^{-3}$ Ha on a $32 \times 32 \times 32$ mesh $(229,376$ degrees of freedom). While the use of adaptive higher-order finite elements will require fewer basis functions than uniform FE, previous studies (Goedecker and Ivanov 1998; Torsti et al. 2006, Bylaska et al. 2009; Lehtovaara et al. 2009; Suryanarayana et al. 2010) suggest that their performance in terms of number of basis functions for a prescribed accuracy may not compare favorably to EFE since they do not incorporate physics-based knowledge (nature of variations, in addition to scale) within the approximation space.

The numerical integration for FE and EFE solutions is performed using tensor-product Gaussian quadrature rules. For EFE computations, the number of integration points are increased until the integration error is below the approximation error. Due to the large values and sharp variations of the enrichment functions in the vicinity of the atomic positions, higher-order quadrature is required in finite elements that are close to the atoms. As 


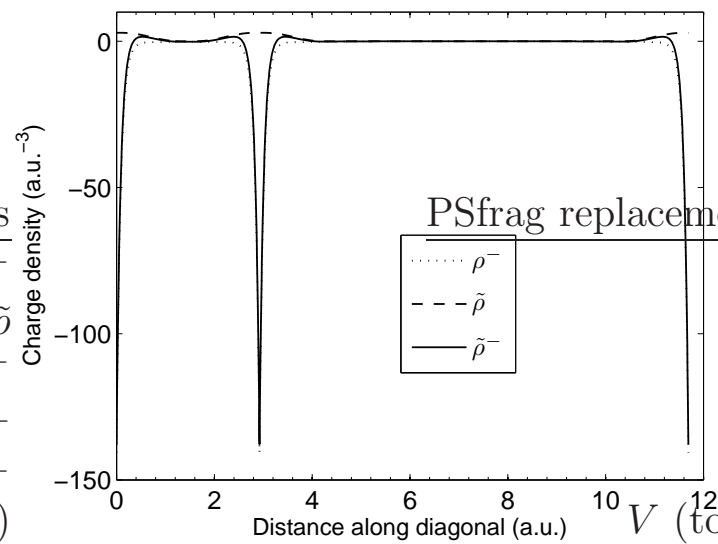

(a)

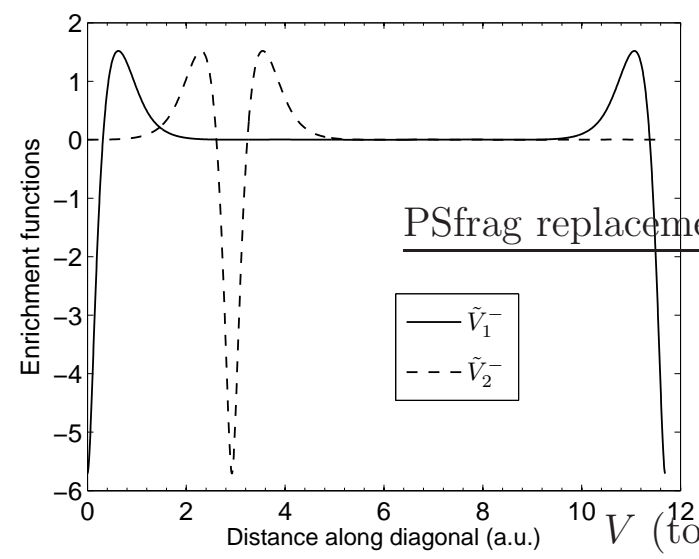

(c)

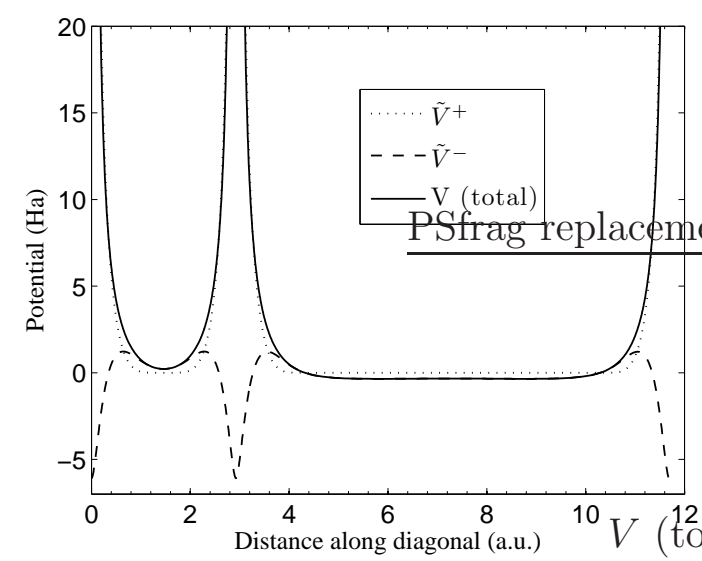

(e)

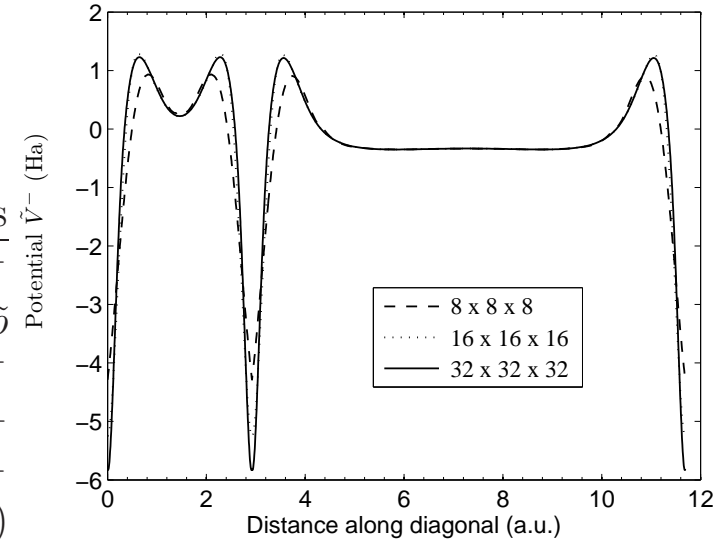

(b)

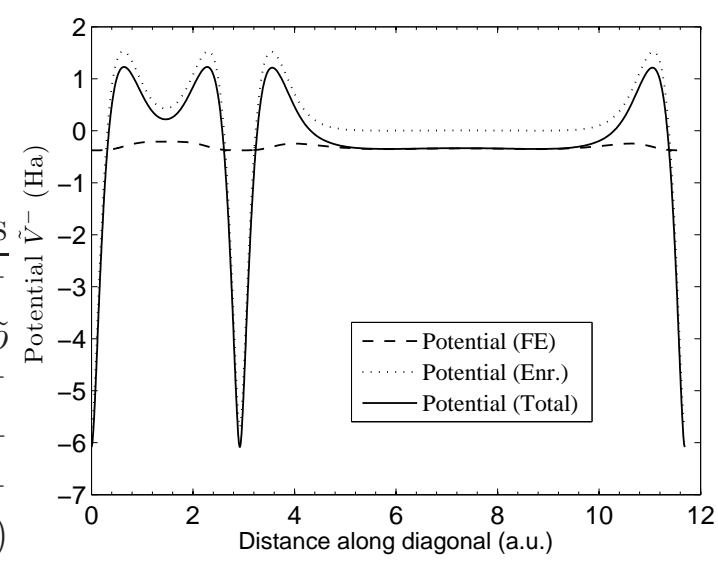

(d)

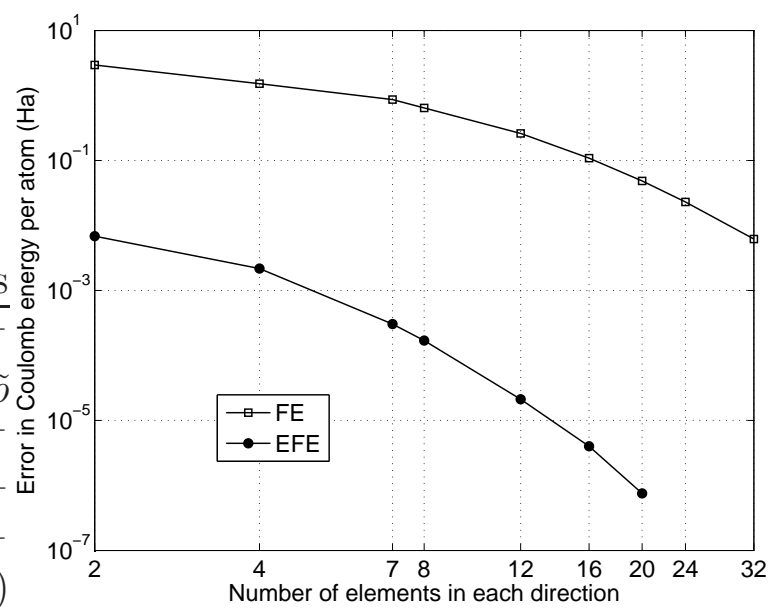

(f)

Figure 7: Finite element (FE) and enriched finite element (EFE) solutions for crystalline diamond. (a) Charge densities; (b) FE solutions; (c) Enrichment functions; (d) EFE solution $(24 \times 24 \times 24$ mesh); (e) Total EFE solution $(24 \times 24 \times 24$ mesh); and (f) Error in Coulomb energy per atom (integral expression). Convergence rate for EFE: meshes 8-12-16 give 5.38 and meshes 12 and 16 yield 5.79. EFE solution on $24 \times 24 \times 24$ mesh is used as reference. 
a result, we use higher-order quadrature in elements with at least one vertex at an atomic position, and a lower-order quadrature rule in all other elements. The reference solution for the Coulomb energy per atom in Figure $7 \mathrm{f}$ is $-75.985203 \mathrm{Ha}$, which is obtained using a $25 \times 25 \times 25$ quadrature rule in elements that are in the vicinity of the atoms and a $15 \times 15 \times 15$ quadrature rule in other elements. The integration scheme we adopt is the simplest rule that provides us with the required accuracy. For better efficiency, a possible approach is to use tetrahedral mesh generation techniques to construct a partition of a finite element with an atom (Coulomb singularity) located at one of its vertices or in its interior. With a graded tetrahedral mesh that is focused towards the atomic position, standard quadrature rules within the tetrahedral elements would suffice to accurately integrate the neutralized charge density and the enrichment functions. Within an EFE method, the tetrahedral mesh so constructed will be solely used for the purpose of numerical integration; the number of degrees of freedom remain unchanged. For the Coulomb singularity, Batcho (2000) and Havu et al. (2004) adopt the Duffy transformation (Duffy 1982) to numerically integrate matrix-elements with $1 / r$ factors. The development of accurate and efficient quadrature schemes in EFE methods is a topical issue at the forefront of current research; recent work in this direction includes a generalization of the Duffy transformation for integrating power singularities (Mousavi and Sukumar 2010). Adaptive integration schemes or quadrature rules that are tailored to the form of the enriched FE basis functions could prove to be more efficient to solve the neutralized Poisson problem. This and related topics are subjects of ongoing research.

\section{Conclusions}

We have presented a linear scaling formulation for the solution of the all-electron Coulomb problem in crystalline solids. The resulting method includes full nuclear potentials, with no smearing approximations, is systematically improvable, and well suited to large-scale quantum mechanical calculations in condensed matter in which the Coulomb potential and energy of a continuous electronic and singular nuclear density are required. Linear scaling is achieved by introducing smooth, strictly local neutralizing densities to render nuclear interactions strictly local, and solving the remaining neutral Poisson problem for the electrons in real space. Rapid variations of the electronic density in the vicinity of the nuclei were efficiently treated using enriched FE methods, with isolated atomic solutions as enrichments. By considering different interaction terms, we derived two equivalent expressions for the Coulomb energy per unit cell-one involving a pointwise evaluation of the neutralized electronic potential, the other requiring the evaluation of a spherical integral. By avoiding pointwise evaluation of the $C^{0} \mathrm{FE}$ solution, the integral expression proved superior in both accuracy and convergence rate with respect to the number of elements. For the Ewald problem, accuracy of order $10^{-8} \mathrm{Ha}$ was realized with enriched FE on a mesh with 5105 degrees of freedom. Comparable accuracy on a uniform FE mesh required 229,376 degrees of

freedom. For the Coulomb energy of diamond, the enriched FE solution with 28,674 degrees of freedom attained an accuracy of $4 \times 10^{-6} \mathrm{Ha}$.

While the calculations here employed a finite element basis, the formulation applies quite generally to any desired basis for the residual Poisson solution and so should prove of wide 
applicability. For example, in the context of high-accuracy Gaussian based calculations, where fast multipole methods become more costly, the present method may provide an attractive alternative for $O(N)$ parallel solution. In such a case, the strictly local polynomial neutralizing functions employed here might be replaced by correspondingly localized Gaussians. Any convenient functional form satisfying the required non-overlap conditions can be employed to equal effect.

\section{Acknowledgments}

This work was performed under the auspices of the U.S. Department of Energy by Lawrence Livermore National Laboratory under Contract DE-AC52-07NA27344. We gratefully acknowledge support from the Laboratory Directed Research and Development Program; the National Science Foundation through contract grant DMS-0811025 to the University of California at Davis; and additional financial support from the UC Lab Fees Research Program.

\section{References}

Arias, T. A. (1999). Multiresolution analysis of electronic structure: Semicardinal and wavelet bases. Rev. Mod. Phys. 71 (1), 267-311.

Babuška, I. and J. M. Melenk (1997). The partition of unity method. Int. J. Numer. Meth. Engng 40, 727-758.

Batcho, P. F. (2000). Computational method for general multicenter electronic structure calculations. Phys. Rev. E 61(6), 7169-7183.

Beck, T. L. (2000). Real-space mesh techniques in density-functional theory. Rev. Mod. Phys. 72(4), 1041-1080.

Blöchl, P. E. (1994). Projector augmented-wave method. Phys. Rev. B 50(24), 1795317979.

Brandt, A. (1977). Multilevel adaptive solutions to boundary-value problems. Math. Comput. 31 (138), 333-390.

Bylaska, E. J., M. Holst, and J. H. Weare (2009). Adaptive finite element method for solving the exact Kohn-Sham equation of density functional theory. J. Chem. Theory Comput. 5(4), 937-948.

Challacombe, M., C. White, and M. Head-Gordon (1997). Periodic boundary conditions and the fast multipole method. J. Chem. Phys. 107(23), 10131-10140.

Duffy, M. G. (1982). Quadrature over a pyramid or cube of integrands with a singularity at a vertex. SIAM J. Numer. Anal. 19(6), 1260-1262.

Ewald, P. P. (1921). Die Berechnung optischer und elektrostatischer Gitterpotentiale. Ann. der Physik 369(3), 253-287.

Fuchs, K. (1935). A quantum mechanical investigation of the cohesive forces of metallic copper. Proc. R. Soc. A 151 (A874), 585-602. 
Genovese, L., T. Deutsch, A. Neelov, S. Goedecker, and G. Beylkin (2006). Efficient solution of Poisson's equation with free boundary conditions. J. Chem. Phys. 125(7), 074105.

Goedecker, S. and O. V. Ivanov (1998). Linear scaling solution of the Coulomb problem using wavelets. Solid State Commun. 105(11), 665-669.

Greengard, L. and V. Rokhlin (1987). A fast algorithm for particle simulations. J. Comput. Phys. 73(2), 325-348.

Havu, P., V. Havu, M. J. Puska, and R. M. Nieminen (2004). Nonequilibrium electron transport in two-dimensional nanostructures modeled using Green's functions and the finite-element method. Phys. Rev. B $69(11), 115325$.

Hohenberg, P. and W. Kohn (1964). Inhomogeneous electron gas. Phys. Rev. 136(3B), B864-B871.

Ihm, J., A. Zunger, and M. L. Cohen (1979). Momentum-space formalism for the total energy of solids. J. Phys. C 12(21), 4409-4422.

Jones, R. O. and O. Gunnarsson (1989). The density functional formalism, its applications and prospects. Rev. Mod. Phys. 61(3), 689-746.

Juselius, J. and D. Sundholm (2007). Parallel implementation of a direct method for calculating electrostatic potentials. J. Chem. Phys. 126(9), 094101.

Kohn, W. and L. J. Sham (1965). Self-consistent equations including exchange and correlation effects. Phys. Rev. 140(4A), 1133-1138.

Kudin, K. N. and G. E. Scuseria (1998). A fast multipole algorithm for the efficient treatment of the Coulomb problem in electronic structure calculations of periodic systems with Gaussian orbitals. Chem. Phys. Lett. 289, 611-616.

Kudin, K. N. and G. E. Scuseria (2000). Linear-scaling density-functional theory with Gaussian orbitals and periodic boundary conditions: Efficient evaluation of energy and forces via the fast multipole method. Phys. Rev. B 61(24), 16440-16453.

Kudin, K. N. and G. E. Scuseria (2004). Revisiting infinite lattice sums with the periodic fast multipole method. J. Chem. Phys. 121(7), 2886-2890.

Kurashige, Y., T. Nakajima, and K. Hirao (2007). Gaussian and finite-element Coulomb method for the fast evaluation of Coulomb integrals. J. Chem. Phys. 126(14), 144106.

Lehtovaara, L., V. Havu, and M. Puska (2009). All-electron density functional theory and time-dependent density functional theory with high-order finite elements. J. Chem. Phys. 131 (5), 054103.

Losilla, S. A., D. Sundholm, and J. Juselius (2010). The direct approach to gravitation and electrostatics method for periodic systems. J. Chem. Phys. 132(2), 024102.

Madelung, E. (1918). Das elektrische Feld in Systemen von regelmäßig angeordneten Punktladungen. Phys. Z. 19, 524.

Melenk, J. M. and I. Babuška (1996). The partition of unity finite element method: Basic theory and applications. Comput. Meth. Appl. Mech. Engrg. 139(1-4), 289-314. 
Merrick, M. P., K. A. Iyer, and T. L. Beck (1995). Multigrid method for electrostatic computations in numerical density-functional theory. J. Phys. Chem. 99(33), 1247812482.

Modine, N. A., G. Zumbach, and E. Kaxiras (1997). Adaptive-coordinate real-space electronic-structure calculations for atoms, molecules, and solids. Phys. Rev. B 55(16), 10289-10301.

Mousavi, S. E. and N. Sukumar (2010). Generalized Duffy transformation for integrating vertex singularities. Comp. Mech. 45(2-3), 127-140.

Murakami, H., V. Sonnad, and E. Clementi (1992). A 3-dimensional finite-element approach towards molecular SCF computations. Int. J. Quantum Chem. 42(4), 785-817.

Nikolaev, A. V. and P. N. Dyachkov (2002). Solution of periodic Poisson's equation and the Hartree-Fock approach for solids with extended electron states: Application to linear augmented plane wave method. Int. J. Quantum Chem. 89(2), 57-85.

Pask, J. E., B. M. Klein, C. Y. Fong, and P. A. Sterne (1999). Real-space local polynomial basis for solid-state electronic-structure calculations: A finite-element approach. Phys. Rev. B 59(19), 12352-12358.

Pask, J. E. and P. A. Sterne (2005a). Finite element methods in ab initio electronic structure calculations. Model. Simul. Mater. Sci. Eng. 13(3), R71-R96.

Pask, J. E. and P. A. Sterne (2005b). Real-space formulation of the electrostatic potential and total energy of solids. Phys. Rev. B 71 (11), 113101.

Pickett, W. E. (1989). Pseudopotential methods in condensed matter applications. Computer Physics Reports 9(3), 115-197.

Rudge, W. E. (1969). Generalized Ewald potential problem. Phys. Rev. 181 (3), 1020-1024.

Schweitzer, M. A. (2003). A parallel multilevel partition of unity method for elliptic partial differential equations. In Lecture Notes in Computational Science and Engineering, Volume 29. Springer, Heidelberg.

Schweitzer, M. A. (2008). Meshfree and Generalized Finite Element Methods. Habilitation, Institute for Numerical Simulation, University of Bonn.

Singh, D. J. and L. Nordstrom (2006). Planewaves, Pseudopotentials, and the LAPW Method (2nd ed.). Springer, New York.

Soler, J. M., E. Artacho, J. D. Gale, A. García, J. Junquera, P. Ordejón, and D. SánchezPortal (2002). The SIESTA method for ab initio order- $N$ materials simulation. $J$. Phys.-Condes. Matter 14(11), 2745-2779.

Strain, M. C., G. E. Scuseria, and M. J. Frisch (1996). Achieving linear scaling for the electronic quantum Coulomb problem. Science 271(5245), 51-53.

Strang, G. and G. J. Fix (1973). An Analysis of the Finite Element Method. Prentice-Hall, Englewood Cliffs.

Stroud, A. H. (1971). Approximate Calculation of Multiple Integrals. Prentice-Hall, Englewood Cliffs. 
Sukumar, N. and J. E. Pask (2009). Classical and enriched finite element formulations for Bloch-periodic boundary conditions. Int. J. Numer. Meth. Engng 777(8), 1121-1138.

Suryanarayana, P., V. Gavini, T. Blesgen, K. Bhattacharya, and M. Ortiz (2010). Non-periodic finite-element formulation of Kohn-Sham density functional theory. J. Mech. Phys. Solids 58(2), 256-280.

Torsti, T., T. Eirola, J. Enkovaara, T. Hakala, P. Havu, V. Havu, T. Höynälänmaa, J. Ignatius, M. Lyly, I. Makkonen, T. T. Rantala, J. Ruokolainen, K. Ruotsalainen, E. Räsänen, H. Saarikoski, and M. J. Puska (2006). Three real-space discretization techniques in electronic structure calculations. phys. stat. sol. (b) 243(5), 1016-1053.

Tsuchida, E. and M. Tsukada (1995). Electronic-structure calculations based on the finiteelement method. Phys. Rev. B 52(8), 5573-5578.

Wang, J. and T. L. Beck (2000). Efficient real-space solution of the Kohn-Sham equations with multiscale techniques. J. Chem. Phys. 112(21), 9223-9228.

Watson, M. A. and K. Hirao (2008). A linear-scaling spectral-element method for computing electrostatic potentials. J. Chem. Phys. 129(18), 184107.

Weinert, M. (1981). Solution of Poisson's equation: Beyond Ewald-type methods. J. Math. Phys. 22(11), 2433-2439.

White, S. R., J. W. Wilkins, and M. P. Teter (1989). Finite-element method for electronicstructure. Phys. Rev. B 39(9), 5819-5833.

Wigner, E. P. and F. Seitz (1933). On the constitution of metallic sodium. Phys. Rev. 43(10), 804-810.

Yamakawa, S. and S. Hyodo (2005). Gaussian finite-element mixed-basis method for electronic structure calculations. Phys. Rev. B 71 (3), 035113.

Zienkiewicz, O. C., R. L. Taylor, and J. Z. Zhu (2005). The Finite Element Method: Its Basis and Fundamentals (Sixth ed.). Elsevier Butterworth-Heinemann. 